\title{
Laser shock microforming of thin metal sheets
}

\author{
J.L. Ocaña *, M. Morales, J.J. García-Ballesteros, J.A. Porro, O. García, C. Molpeceres \\ Centro Láser UPM, Universidad Politécnica de Madrid, Campus Sur UPM, Edificio La Arboleda, Ctra. de Valencia km 7.3, 28031 Madrid, Spain
}

Keywords:

Laser microforming

Forming mechanisms

Numerical modeling

Experimental validation

\begin{abstract}
A B S T R A C T
Continuous and long-pulse lasers have been used for the forming of metal sheets in macroscopic mechanical applications. However, for the manufacturing of micro-electromechanical systems (MEMS), the applicability of such type of lasers is limited by the long-relaxation-time of the thermal fields responsible for the forming phenomena. As a consequence of such slow relaxation, the final sheet deformation state is attained only after a certain time, what makes the generated internal residual stress fields more dependent on ambient conditions and might make difficult the subsequent assembly process from the point of view of residual stresses due to adjustment.

The use of ns laser pulses provides a suitable parameter matching for the laser forming of an important range of sheet components used in MEMS that, preserving the short interaction time scale required for the predominantly mechanic (shock) induction of deformation residual stresses, allows for the successful processing of components in a medium range of miniaturization, particularly important according to its frequent use in such systems.

In the present paper, a discussion is presented on the physics of laser shock microforming and the influence of the different effects on the net bending angle. The experimental setup used for the experiments, sample fabrication and experimental results of influence of number of laser pulses on the net bending angle are also presented.
\end{abstract}

\section{Introduction}

The increasing demands in MEMS fabrication are leading to new requirements in production technology [1-3]. Especially packaging and assembly require high accuracy in positioning and high reproducibility in combination with low production costs.

Accurate positioning of smallest components represents an upto-date key assignment in micro-manufacturing and initial assembly with widened tolerances complemented by a final micro-adjusting has proven to be a time and cost efficient alternative [2].

Provided that as mounted micro components are typically difficult to access and highly sensitive to mechanical forces and impacts, contact-free laser adjustment processes offer a great potential for accurate manipulation of micro devices.

Laser shock forming is a non-thermal laser forming method using the shock wave induced by laser irradiation to modify the curvature of the target $[4,5]$. It has the advantages of laser thermal forming (non-contact, tool-free and high efficiency and precision), but its non-thermal character allows the preservation or even improvement of material properties through the induction of compressive residual stress over the target surface, a feature enabling an improved resistance of shaped metal to resist corrosion and fatigue.

In this paper, the physics of laser shock microforming of thin metallic films is studied along with the influence of process parameters on the net bending angle. The experimental setup used for the validation experiments, sample fabrication details and experimental results on the practical conformability of test components are also presented. For conceptual simplicity reasons a simple forming geometry (one-side pinned flat metal stripe) has been chosen for the presented analysis (see Fig. 1).

\section{Physics of laser shock microforming numerical modeling}

Laser shock microforming ( $\mathrm{LS} \mu \mathrm{F}$ ) is based on the application of a high intensity pulsed laser beam $\left(I>10^{9} \mathrm{~W} / \mathrm{cm}^{2} ; \tau<50 \mathrm{~ns}\right)$ on a metallic target forcing a sudden vaporization of its surface into a high temperature plasma that immediately develops inducing a shock wave propagating into the material.

In laser-induced shock processes, the material is stressed and deformed in a dynamic way, with strain rates exceeding $10^{6} \mathrm{~s}^{-1}$. 


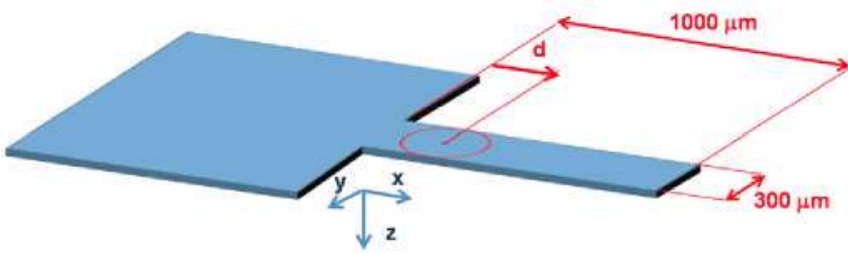

Fig. 1. Schematic diagram of thin film and the flat metal stripe and definition of distance $d$ (distance to thin film/flat metal stripe union).

The plastic deformation induced by the shock wave generates a residual stress distribution in the material. Stress in the direction of the stripe $\left(\sigma_{x x}\right)$ is compressive at the incident surface and tensile at the rear surface (see Fig. 2). This stress distribution produces a local bending $\left(\beta_{s}\right)$ in the direction of the laser beam.

Contrarily, the application of the laser pulse at a given distance $\mathrm{d}$ from the clamping generates a bending moment associate to the laser pulse energy. This bending moment can produce plastic deformation near the clamping so that, in this case, stress in the stripe direction $\left(\sigma_{x x}\right)$ is tensile at the incident surface and compressive at the rear surface (see Fig. 2 ). This stress distribution tends to produce a drag bending $\left(\beta_{\mathrm{b}}\right)$ in the opposite direction of the laser beam (see Fig. 2).

The combination of these two effects produces the net bending angle $(\alpha)$, determined for small angles as the ratio between the apparent bar end displacement to the bar length (see again Fig. 2). This net bending angle can be optimized as a function of the laser characteristics, namely pulse duration, wavelength and energy and other process parameters, namely the laser spot radius, spot position and the nature and thickness of the metal stripe.

The authors have developed a calculational system to study laser shock microforming [5-7]. It consists of two principal modules conceived for the analysis of plasma evolution (shock waves generation) and induced residual stresses (shock wave evolution and material plastic deformation) under two different but complementary approaches [7]. Provided that the effects to be analyzed are strongly non linear as a conference of the leading role of material plastic deformations, the influence of the different process parameters (laser energy and laser spot position) on net bending angle have been studied with the developed numerical model.

In Fig. 3, the numerical study of net bending angle of the metal sheet $(\alpha)$ as a function of the laser energy pulse is displayed together with the two components, $\beta_{\mathrm{s}}$ and $\beta_{\mathrm{b}}$. The results show that, after a critical value of pulse energy, the bending angle $\left(\beta_{\mathrm{b}}\right)$ produced by the bending moment starts to have an important
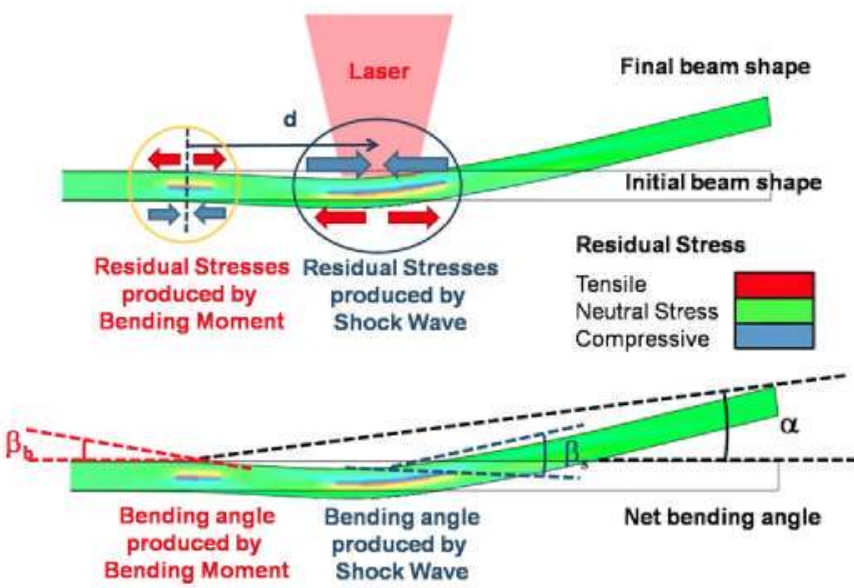

Fig. 2. Schematic diagram of thin film deformation and stresses and definition of bending angles.

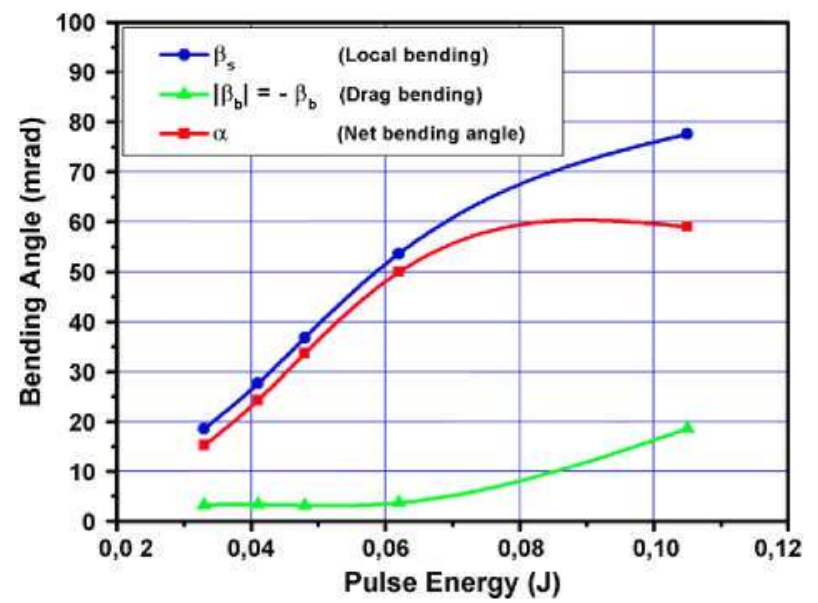

Fig. 3. Net bending angle, local bending and drag bending of reference thin sheet specimens subject to 10 ns laser pulses irradiation at $d=L / 3$ as a function of laser pulse energy.

deleterious effect on the net bending angle $(\alpha)$ : The higher pressure at the laser incidence region starts to saturate the residual stress distribution while the drag bending moment starts to be higher in value.

On the other hand, the numerical study of the net bending angle of the metal sheet as a function of the laser spot position, $d$, (see Fig. 4) shows that, as a consequence of the combination of the two described main effects, a maximum is obtained when the laser spot is applied at a given distance $d(d \cong L / 3$ in this case). The residual stress distribution produced by shock waves is the same in all these cases, but when the laser is near the bulk material the bending angle is reduced because of the reduced bending moment (linearly increasing with $d$ ).

\section{Experimental}

The validity of the assumption and the results presented in the theoretical section have been experimentally validated. For this purpose, a set of micro-samples reproducing the conditions of the analyzed specimens were produced and the theoretical results referred to overall bending angle $(\alpha)$ were checked against the corresponding experimental values. In the next paragraphs the relevant aspects of the followed experimental procedure are provided.

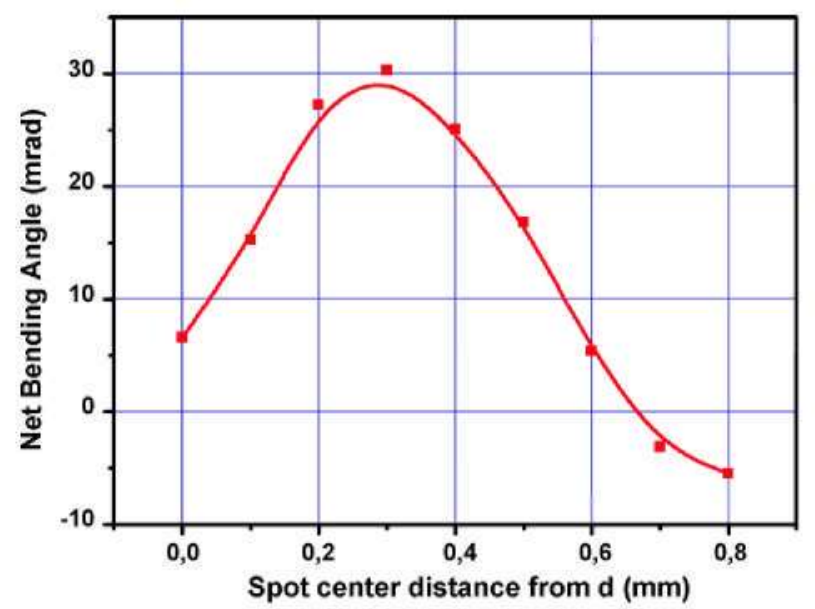

Fig. 4. Net bending angle of reference thin sheet specimens subject to $0.048 \mathrm{~J}, 10 \mathrm{~ns}$ laser pulses irradiation as a function of spot center distance from base, $d$. 


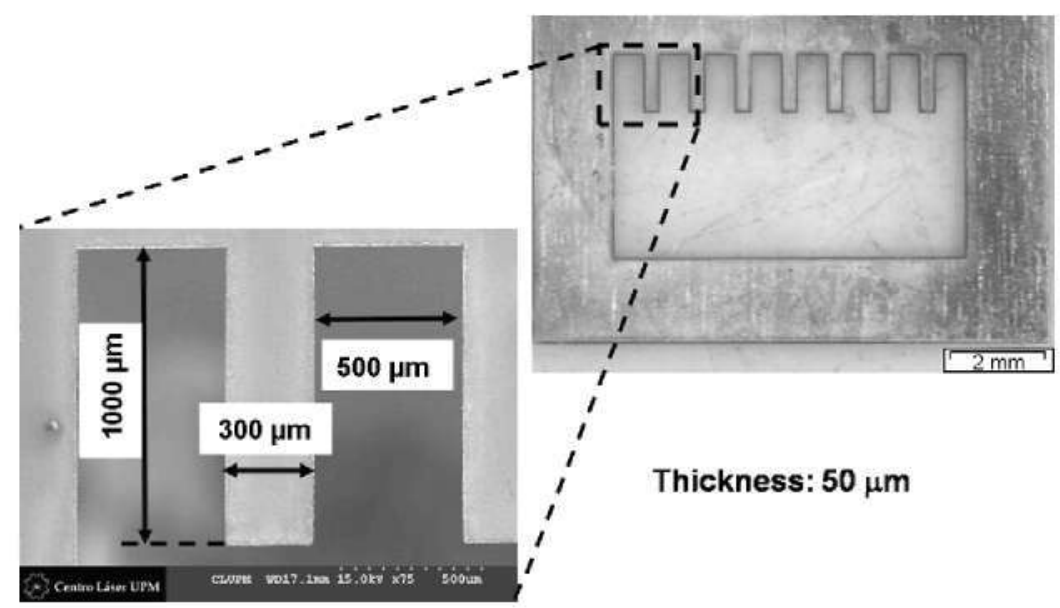

Fig. 5. Photograph of flat metal stripe set and SEM photograph of individual flat metal stripe with dimensions.

\subsection{Fabrication of micro-samples}

A laser micromachining workstation equipped with a $\mathrm{Nd}: \mathrm{YVO}_{4}$ (Spectra Physics HIPPO) working at UV (355 nm) was used for the manufacturing of the specimens [8].

The specimens considered in this paper are in Stainless Steel AISI 304 ( $\mathrm{Fe} 72 \%, \mathrm{Cr} 18 \%, \mathrm{Ni} 10 \%$ ) and were machined in sets containing several metal stripes from a larger metal sheet. Fig. 5 shows a photograph of a sample set and a detail of an individual stripe.

\subsection{Laser shock microforming experimental setup}

The practical irradiation system used in the experiments has been previously reported by the authors in a former paper [5] and is essentially consisting of a Q-switched laser with emission at $1064 \mathrm{~nm}$ and providing a maximum energy of $2 \mathrm{~J} /$ pulse in a FWHM pulse length of $10 \mathrm{~ns}$ coupled through a conventional optical path to $\mathrm{XY}$ motion stage allowing the precise positioning of the target. Beam focusing to the required spot size is performed by means of an adjustable focus optics within intensity limits allowed by the absence of air dielectric breakdown. In order to control this limitation and select the appropriate beam energy level for each particular experiment, a variable diameter mask is used.

\subsection{Experimental results}

According to the methodology defined above, the effect of the application of high intensity laser pulses to the bending of metal

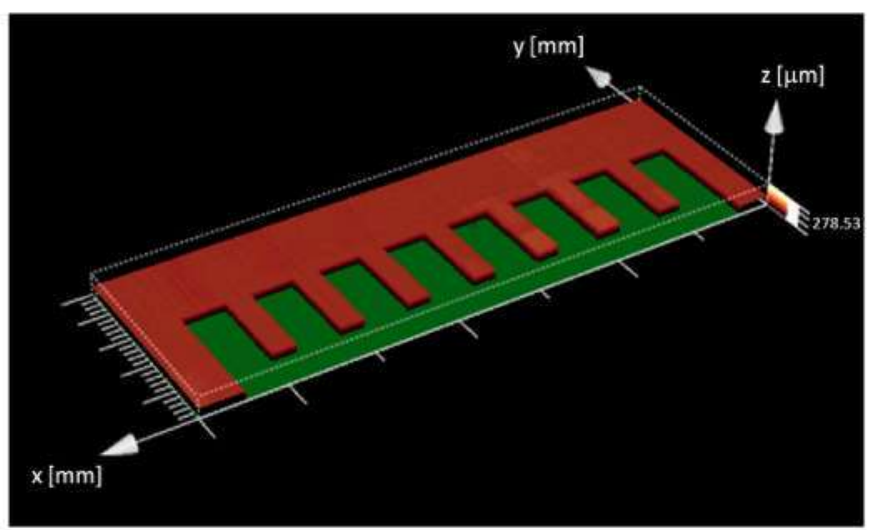

Fig. 6. Confocal microscopy image of sample. stripes was experimentally tested and compared to predictive numerical results obtained by the described theoretical model. Table 1 shows the relevant experimental conditions, the contrast parameter being the metal stripe deformation for a variable distance $d$.

The deformation profiles were assessed by means of a LEICA ICM 1000 ( $\lambda=635 \mathrm{~nm})$ confocal laser scanning microscope. In Fig. 6 a sample confocal image of the deformed specimens after treatment with different parametric values $(d=-78 \mu \mathrm{m}, 150 \mu \mathrm{m}$ and $820 \mu \mathrm{m}$ ) is shown and in Fig. 7 a comparison of the bending

Table 1

Experimental laser shock microforming parameters.

\begin{tabular}{ll}
\hline Nd:YAG laser wavelength $(\mathrm{nm})$ & 1064 \\
Energy per pulse $(\mathrm{J})$ & 1.651 \\
Pulse length FWHM (ns) & 9 \\
Beam radius $(\mathrm{mm})$ & 14 \\
Mask radius $(\mu \mathrm{m})$ & 750 \\
Energy per pulse (after mask) (mJ) & 57 \\
Energy per pulse (after mask and lens) $(\mathrm{mJ})$ & 54 \\
Spot radius $(\mu \mathrm{m})$ & 150 \\
Confining layer & Air \\
Thin sheet material & AlS1 304 \\
Thin sheet thickness $(\mu \mathrm{m})$ & 50 \\
Spot center position, $d(\mu \mathrm{m})$ & 150
\end{tabular}

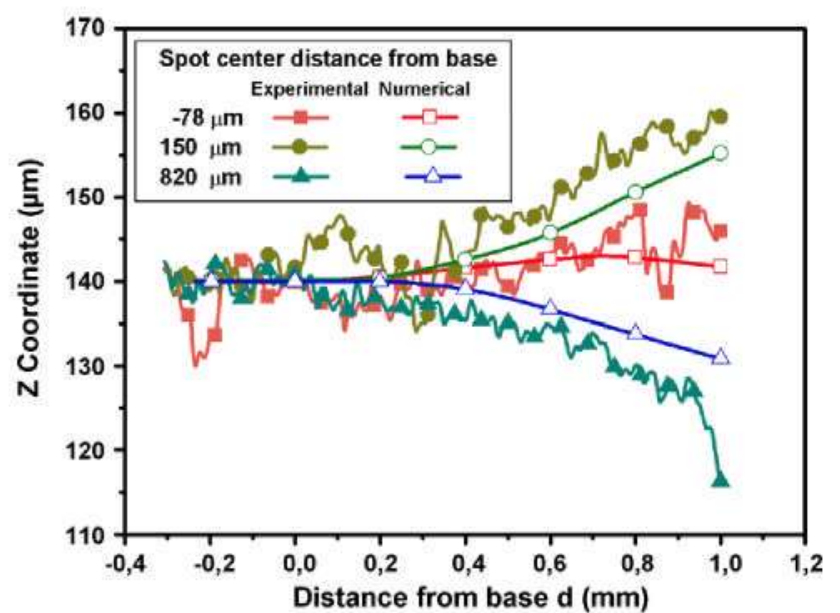

Fig. 7. Experimental and numerical simulation profiles obtained for different laser spot positions. 

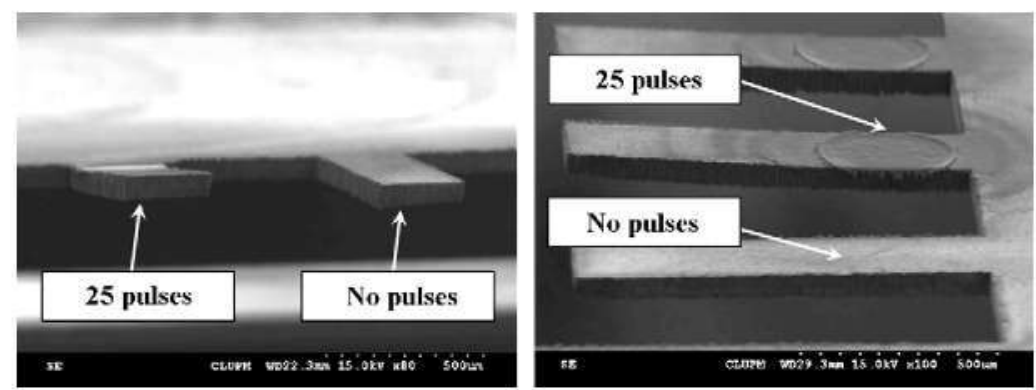

Fig. 8. Scanning electron microscope (SEM) photograph of the flat metal stripe after 25 pulses and an untreated flat metal stripe.

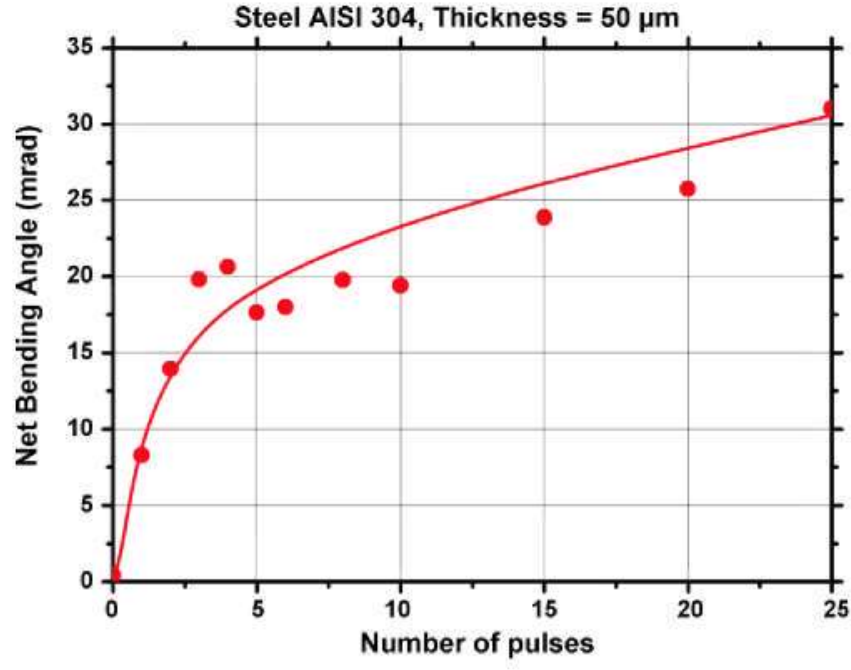

Fig. 9. Influence of number of laser pulses on net bending angle.

profiles of the stripes compared to the theoretical predictions is displayed, the contrast between theoretical and experimental results being quite satisfactory.

Additionally to the described theory-experiment validation procedure, and in order to assess the effect of multiple laser pulses on the final conformability of the considered specimens, the stripes irradiation by different number of laser pulses (up to 25) was accomplished, In Fig. 8 the SEM view of a particular specimen irradiated with 25 pulses is shown in comparison to a nonirradiated sample, and in Fig. 9 a plot is provided showing the progressive effect of an increasing number of applied pulses in the conformation final angle of a typical specimen: the clear effect is shown of the practical conformability of the metal and the saturation effect originated by the progressive influence of residual stresses induced by previous pulses.

\section{Discussion}

According to the previously presented results, the practical feasibility of the shock laser microforming process developed on the basis of ns range $\mathrm{GW} / \mathrm{cm}^{2}$ lasers has been demonstrated, not only from a practical point of view but in close consistency with the relevant expected physical effects following high intensity laser interaction, very suitably addressed by the developed calculational model.

Further to the validation of this model, the authors have proven the practical feasibility of finite angle microforming by sequential accumulation of laser pulses on a given specimen, then opening the way for a practical technology implementation.

\section{Acknowledgements}

Work partly supported by Spanish MEC Projects PSE0204002006-1, PSE020400-2007-2 and CIT0205002005-11.

\section{References}

[1] F. Vollertsen, Z. Hu, H. Schulze Niehoff, C. Theiler, J. Mater. Process. Technol. 151 (2004) 70 .

[2] M. Dirscherl, G. Esser, M. Schmidt, J. Laser Micro/Nanoeng. 1 (2006) 50.

[3] H.W. Jeong, S. Hata, A. Shimokohbe, J. Microelectromech. Syst. 12 (2003) 42.

[4] W. Zhang, Y.L. Yao, I.C. Noyan, Trans. ASME 126 (2004) 10.

[5] J.L. Ocaña, M. Morales, C. Molpeceres, O. García, J.A. Porro, J.J. García-Ballesteros, Appl. Surf. Sci. 254 (2007) 997.

[6] J.L. Ocaña, C. Molpeceres, M. Morales, A. García-Beltrán, in: C.R. Phipps, M. Niino (Eds.), High-Power Laser Ablation II SPIE Proceedings, vol. 3885, 2000, p. 252.

[7] J.L. Ocaña, M. Morales, C. Molpeceres, J. Torres, Appl. Surf. Sci. 238 (2004) 242.

[8] C. Molpeceres, S. Lauzurica, J.J. García-Ballesteros, M. Morales, J.L. Ocaña, Microelectron. Eng. 84 (2007) 1337. 